\title{
Marasmane Sesquiterpenes Isolated from Russula foetens
}

\author{
Xing-Na Wang, Jian-Hua Shen, Jian-Chang Du, Ji-Kai Liu
}

Received: June 1, 2006 / Accepted: October 10, 2006

(C) Japan Antibiotics Research Association

\begin{abstract}
One lactone marasmane sesquiterpene named $8 \alpha, 13$-dihydroxy-marasm-5-oic acid $\gamma$-lactone (1) and one unsaturated marasmane sesquiterpene named 13-hydroxymarasm-7(8)-en-5-methoxy $\gamma$-acetal (2) together with a known compound: $7 \alpha, 8 \alpha, 13$-trihydroxy-marasm-5-oic acid $\gamma$-lactone (3) were isolated from the fruiting bodies of Russula foetens. Their structures were established on the basis of spectral methods (MS, IR, 1D and 2D NMR experiments).
\end{abstract}

Keywords Russula foetens, marasmane sesquiterpenes, structure determination

Members of the genus Russula are important symbionts, forming mycorrhiza with higher plants which explains in some cases their preference for growing among certain kinds of trees. The genus is one of the largest in Agaricales and is distributed worldwide; more than 100 species are reported to grow in China where mixed forests are their typical habitat. While secondary metabolites occurring in the fruiting bodies of Lactarius species have been well investigated, the Russula mushrooms have received less attention, notwithstanding the large number of existing species [1]. The fungal subdivision Basidiomycotina produces many toxic sesquiterpenes derived from the protoilludane skeleton. This skeleton is transformed and rearranged to a multitude of compounds. Fungal sesquiterpenes formed via the humulane-protoilludane biosynthetic pathway are characteristic for the subdivision

X. N. Wang, J. H. Shen: Shanghai Institute of Materia Medica, the Chinese Academy of Sciences, Shanghai 201203, P. R. China

J. C. Du: Institute of Health Sciences, Shanghai, Institutes for Biological Sciences, the Chinese Academy of Sciences, Shanghai, 200025, P. R. China
Basidiomycotina [2]. Sesquiterpenes possessing the marasmane skeleton have been known for more than 50 years [3]. The marasmane sesquiterpenes often have antibiotic activities [4 8].

From the higher fungus Russula foetens we had isolated two marasmane sesquiterpenes: lactapiperanol A [9] and lactapiperanol E [10]. In this paper, we will report the isolation of another three compounds (1 $\mathbf{3})$, of which 1 and $\mathbf{2}$ are new.

The fruiting bodies of $R$. foetens were collected at Ailao Mountain of Yunnan Province, China, in July, 2004 and identified by Prof. Mu Zang, Kunming Institute of Botany, the Chinese Academy of Sciences. The voucher specimen was deposited at the Herbarium of Kunming Institute of Botany, the Chinese Academy of Sciences. The air-dried fruiting bodies of $R$. foetens $(1 \mathrm{~kg})$ were crushed and extracted with $\mathrm{CHCl}_{3} / \mathrm{MeOH}(1 / 1, \mathrm{v} / \mathrm{v})$ three times at room temperature. The combined extracts were concentrated in vacuo to give a syrup $(70 \mathrm{~g})$ which was chromatographed on a silica gel $(1500 \mathrm{~g})$ column, using a gradient elution with $\mathrm{CHCl}_{3} / \mathrm{MeOH}(100: 0$ to $0: 100, \mathrm{v} / \mathrm{v})$ to afford six fractions. Fr. $2\left(\mathrm{CHCl}_{3} / \mathrm{MeOH}, 95: 5\right)$ was subjected to silica gel chromatography using a gradient elution with $\mathrm{CHCl}_{3}$ /acetone (100:0 to $\left.50: 50\right)$ and four fractions were obtained. Further separation of Fr. $2.1\left(\mathrm{CHCl}_{3} /\right.$ acetone, $98: 2)$ over silica gel with petroleum ether/acetone $(100: 1)$ afforded compound 2. Fr. $2.4\left(\mathrm{CHCl}_{3} /\right.$ acetone, $\left.50: 50\right)$ was purified over silica gel with $\mathrm{CHCl}_{3} /$ acetone $(50: 50)$ repeatedly to give compound 3. Fr. $3\left(\mathrm{CHCl}_{3} / \mathrm{MeOH}\right.$, $90: 10)$ was subjected to Sephadex LH-20 to get three fractions. Fraction 3.2 was separated by preparative thin

J. K. Liu (Corresponding author): Kunming Institute of Botany, the Chinese Academy of Sciences, Kunming 650204, P. R. China, E-mail: jkliu@mail.kib.ac.cn 
Table 1 The ${ }^{1} \mathrm{H},{ }^{13} \mathrm{C}$-NMR assignments, HMBC and NOESY correlations of compound 1

\begin{tabular}{|c|c|c|c|c|}
\hline Position & $\delta_{\mathrm{H}}$ & $\mathrm{HMBC}$ & NOESY & $\delta_{\mathrm{C}}$ \\
\hline $1 \mathrm{a}$ & $1.68(\mathrm{dd}, J=14.0,6.8)$ & & & $41.8(\mathrm{t})$ \\
\hline $1 b$ & $1.47(\mathrm{dd}, J=14.0,13.4)$ & & & \\
\hline 2 & $2.63(\mathrm{ddd}, J=13.4,6.8,6.7)$ & C-8 & $\mathrm{H}_{3}-14$ & 45.2 (d) \\
\hline 3 & / & & & $28.4(\mathrm{~s})$ \\
\hline $4 a$ & $1.36(\mathrm{~d}, J=4.2)$ & & & $29.0(t)$ \\
\hline $4 b$ & $0.96(d, J=4.2)$ & & $\mathrm{H}-7$ & \\
\hline 5 & l & & & 177.9 (s) \\
\hline 6 & l & & & 29.5 (s) \\
\hline 7 & $2.40(\mathrm{ddd}, J=9.3,9.2,9.2)$ & & $\mathrm{H}-4 \mathrm{~b}$ & 43.8 (d) \\
\hline 8 & 3.27 (dd, J=9.9, 10.2) & C-13, C-10 & $\mathrm{H}_{3}-15, \mathrm{H}-13 \mathrm{a}$ & 73.6 (d) \\
\hline 9 & $1.59(\mathrm{~m})$ & & & 44.8 (d) \\
\hline $10 a$ & $1.66(\mathrm{dd}, J=14.3,1.0)$ & & & $44.7(\mathrm{t})$ \\
\hline $10 \mathrm{~b}$ & $1.58(\mathrm{dd}, J=14.3,7.3)$ & & & \\
\hline 11 & / & & & 37.0 (s) \\
\hline 12 & 1.29 (s) & $\mathrm{C}-2, \mathrm{C}-4, \mathrm{C}-6$ & & $17.4(q)$ \\
\hline $13 a$ & $4.18(\mathrm{dd}, J=7.3,9.3)$ & & $\mathrm{H}-8$ & $71.5(t)$ \\
\hline $13 b$ & $4.71(t, J=9.3)$ & C-6 & & \\
\hline 14 & $1.26(\mathrm{~s})$ & C-1, C-10 & $\mathrm{H}-2$ & $31.8(q)$ \\
\hline 15 & 1.05 (s) & C-1, C-10 & $\mathrm{H}-8$ & $32.4(q)$ \\
\hline
\end{tabular}

layer chromatography with $\mathrm{CHCl}_{3} / \mathrm{MeOH}(100: 5)$ to afford compound 1 with strong fluorescence at $365 \mathrm{~nm}$.

Compound 1 was isolated as oil. The molecular formula of compound 1 was determined to be $\mathrm{C}_{15} \mathrm{H}_{22} \mathrm{O}_{3}$ on the basis of HR-EI-MS [ $\mathrm{m} / \mathrm{z} 250.1574$ (Calcd. 250.1569)]. The IR spectrum of 1 showed a broad hydroxyl band at $3423 \mathrm{~cm}^{-1}$ and a lactonic carbonyl absorption at $1758 \mathrm{~cm}^{-1}$. These features were confirmed by ${ }^{13} \mathrm{C}$-NMR which exhibited signals for a $\mathrm{C}$ atom substituted with hydroxyl oxygen atom $(\delta 73.6, \mathrm{C}-8, \mathrm{~d})$ and a quaternary carbon $(\delta 177.9, \mathrm{C}-5, \mathrm{~s})$. Three methyl groups $(\delta 1.05,1.26$ and 1.29 respectively, each $3 \mathrm{H}, \mathrm{s}$ ) were shown in the ${ }^{1} \mathrm{H}-\mathrm{NMR}$ spectrum of $\mathbf{1}$. The molecular formula $\mathrm{C}_{15} \mathrm{H}_{22} \mathrm{O}_{3}$ suggested $\mathbf{1}$ had five degrees of unsaturation. Each of the two H-4 protons showed a doublet in the ${ }^{1} \mathrm{H}-\mathrm{NMR}$ spectrum with a characteristic coupling constant of $J=$ about $5 \mathrm{~Hz}(\delta 1.36, \mathrm{H}-4 \mathrm{a}, \mathrm{d}$, $J=4.2 \mathrm{~Hz} ; \delta 0.96, \mathrm{H}-4 \mathrm{~b}, \mathrm{~d}, J=4.2 \mathrm{~Hz}$ ) allowed us to assign the marasmane skeleton, but not lactarane skeleton, to compound $\mathbf{1}$. The ${ }^{1} \mathrm{H}-{ }^{1} \mathrm{H}$ COSY spectrum of $\mathbf{1}$ exhibited the connections between H-8 and H-9, H-9 and H-2, H-8 and $\mathrm{H}-7, \mathrm{H}-7$ and $\mathrm{H}-13_{\mathrm{ab}}$ clearly. The HMBC spectrum shown in Table 1 gave the structure of $\mathbf{1}$.

The NOESY cross peaks were observed between H-8 and $\mathrm{H}_{3}-15, \mathrm{H}-8$ and H-13a, H-7 and H-4b, H-2 and $\mathrm{H}_{3}-14$. The coupling constant $(\mathrm{d}, J=10.2 \mathrm{~Hz}$ ) for H-8 and H-9 suggested the hydroxyl group at C-8 had trans configuration. According to all the information, we could draw the stereo structure of compound $\mathbf{1}$.

Compound $\mathbf{2}$ was isolated from the very small polar fraction. Its HR-EI-MS with the molecular ion peak at $\mathrm{m} / \mathrm{z} 248.1769$ (calcd. 248.1776) was consistent with the molecular formula $\mathrm{C}_{16} \mathrm{H}_{24} \mathrm{O}_{2}$ which suggested a sesquiterpene skeleton with 5 degrees of unsaturation. The IR spectrum of $\mathbf{2}$ showed no hydroxyl group existed. From the ${ }^{13} \mathrm{C}$-NMR and ${ }^{1} \mathrm{H}-\mathrm{NMR}$, we can see signals of one methoxy group $\left(\delta_{\mathrm{C}} 54.5, \delta_{\mathrm{H}} 3.34,3 \mathrm{H}\right)$, three methyl groups $\left(\delta_{\mathrm{C}} 32.0, \delta_{\mathrm{H}} 0.99,3 \mathrm{H} ; \delta_{\mathrm{C}} 31.9, \delta_{\mathrm{H}} 1.01,3 \mathrm{H} ; \delta_{\mathrm{C}} 21.3, \delta_{\mathrm{H}}\right.$ $1.20,3 \mathrm{H})$, one oxygenated methylene $\left(\delta_{\mathrm{C}} 69.0, \delta_{\mathrm{H}} 4.55\right.$, $2 \mathrm{H})$, one methylene with two doublets in ${ }^{1} \mathrm{H}-\mathrm{NMR}\left(\delta_{\mathrm{C}}\right.$ $26.1, \delta_{\mathrm{Ha}} 0.77$ and $\left.\delta_{\mathrm{Hb}} 0.93,2 \mathrm{H}\right)$, two methylenes $\left(\delta_{\mathrm{C}} 44.2\right.$, $\delta_{\mathrm{Ha}} 1.51$ and $\delta_{\mathrm{Hb}} 1.31,2 \mathrm{H} ; \delta_{\mathrm{C}} 48.2, \delta_{\mathrm{Ha}} 1.78$ and $\delta_{\mathrm{Hb}} 1.33$, $2 \mathrm{H})$, one acetal group $\left(\delta_{\mathrm{C}} 109.0, \delta_{\mathrm{H}} 4.80,1 \mathrm{H}\right)$, one unsaturated methine $\left(\delta_{\mathrm{C}} 115.2, \delta_{\mathrm{H}} 4.84,1 \mathrm{H}\right)$, two methines $\left(\delta_{\mathrm{C}} 42.4, \delta_{\mathrm{H}} 2.40,1 \mathrm{H} ; \delta_{\mathrm{C}} 39.1, \delta_{\mathrm{H}} 2.45,1 \mathrm{H}\right)$, one unsaturated quaternary carbon $\left(\delta_{\mathrm{C}} 139.1\right)$. The above spectral data suggested compound $\mathbf{2}$ was also a marasmane sesquiterpenoid with a methoxy group. The $\mathrm{C}-\mathrm{H}$ longrange correlations observed in HMBC spectrum (Table 2) confirmed the structure of compound 2. Its stereo structure was determined by NOESY spectrum (Table 2).

The spectral data and physical properties of $7 \alpha, 8 \alpha, 13$ trihydroxy-marasm-5-oic-acid $\gamma$-lactone (3) were identical 
Table $2{ }^{1} \mathrm{H},{ }^{13} \mathrm{C}-\mathrm{NMR}$ data, HMBC and NOESY correlations of compound 2

\begin{tabular}{|c|c|c|c|c|}
\hline Position & $\delta_{\mathrm{H}}$ & $\mathrm{HMBC}$ & NOESY & $\delta_{\mathrm{C}}$ \\
\hline $1 a$ & $1.51(\mathrm{~m})$ & & & $44.2(t)$ \\
\hline $1 b$ & $1.31(\mathrm{dd}, J=5.2,12.1)$ & & & \\
\hline 2 & $2.40(\mathrm{~m})$ & $\mathrm{C}-8$ & & 42.4 (d) \\
\hline 3 & l & & & 34.5 (s) \\
\hline $4 a$ & $0.77(d, J=3.7)$ & & & $26.1(\mathrm{t})$ \\
\hline $4 b$ & $0.93(d, J=3.7)$ & & & \\
\hline 5 & 4.80 (s) & & $\mathrm{H}_{3}-12$ & 109.0 (d) \\
\hline 6 & l & & & 24.9 (s) \\
\hline 7 & l & & & 139.1 (s) \\
\hline 8 & $4.84(\mathrm{~m})$ & $C-10, C-13$ & & 115.2 (d) \\
\hline 9 & $2.45(\mathrm{~m})$ & & & 39.1 (d) \\
\hline $10 a$ & $1.78(d d, J=13.3,6.5)$ & & & $48.2(\mathrm{t})$ \\
\hline $10 b$ & $1.33(\mathrm{dd}, J=13.3,10.3)$ & & & \\
\hline 11 & l & & & $37.4(s)$ \\
\hline 12 & 1.20 (s) & $\mathrm{C}-2, \mathrm{C}-4, \mathrm{C}-6$ & $\mathrm{H}-5$ & $21.3(q)$ \\
\hline $13 a$ & $4.55(\mathrm{~m})$ & & & $69.0(\mathrm{t})$ \\
\hline \multicolumn{5}{|l|}{$13 b$} \\
\hline 14 & 1.01 (s) & $\mathrm{C}-1, \mathrm{C}-10$ & & $31.9(q)$ \\
\hline 15 & 0.99 (s) & $\mathrm{C}-1, \mathrm{C}-10$ & & $32.0(q)$ \\
\hline $\mathrm{OCH}_{3}$ & $3.34(\mathrm{~s})$ & $C-5$ & & $54.5(q)$ \\
\hline
\end{tabular}



1

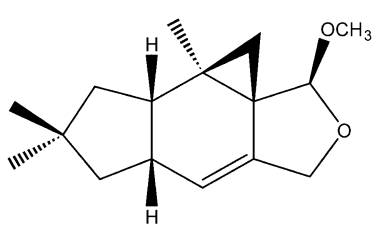

2

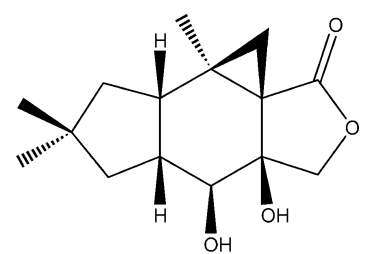

3

Fig. 1 Structures of the three marasmane compounds isolated from Russula foetens.

with the data previously reported [11].

$8 \alpha, 13$-dihydroxy-marasm-5-oic acid $\gamma$-lactone (1). $[\alpha]_{\mathrm{D}}^{25}=+42.2\left(c 0.005, \mathrm{CH}_{2} \mathrm{Cl}_{2}\right)$. EI-MS $m / z$ (rel. int.): 250 $\left([\mathrm{M}]^{+}, 15\right), 232\left(\left[\mathrm{M}-\mathrm{H}_{2} \mathrm{O}\right]^{+}, 17\right), 217\left[\mathrm{M}-\mathrm{H}_{2} \mathrm{O}-\mathrm{CH}_{3}\right]^{+}$, (40), 135 (100). IR (KBr): $v=3423,2925,2867,1758 .{ }^{1} \mathrm{H}$ NMR $\left(400 \mathrm{MHz}, \mathrm{CDCl}_{3}\right)$ and ${ }^{13} \mathrm{C} \mathrm{NMR}\left(100 \mathrm{M}, \mathrm{CDCl}_{3}\right)$ : Table 1.

13-Hydroxy-marasm-7(8)-en-5-methoxy $\gamma$-acetal (2). $[\alpha]_{\mathrm{D}}^{25}=+50.1\left(c 0.003, \mathrm{CH}_{2} \mathrm{Cl}_{2}\right)$. EI-MS $m / z$ (rel. int.): 248 (15), 233 (41), 216 (100), 201 (18), 188 (17), 145 (20), 133 (22). ${ }^{1} \mathrm{H}$ NMR (400 MHz, $\left.\mathrm{CDCl}_{3}\right)$ and ${ }^{13} \mathrm{C}$ NMR $(100 \mathrm{MHz}$, $\mathrm{CDCl}_{3}$ ): Table 2 .

\section{References}

1. Vidari G, Che ZL, Garlaschelli L. New nardosinane and aristolane sesquiterpenes from the fruiting bodies of Russula lepida. Tetrahedron Lett 39: 6073-6076 (1998)

2. Wolf-Rainer A. Bioactive sesquiterpenes produced by fungi: are they useful for humans as well. Cur Med Chem 8: 583-606 (2001)

3. Kavanagh F, Hervey A, Robbins WJ. Antibiotic substances from basidiomycetes: IV. Marasmius conigenus. Proc Natl Acad Sci USA 35: 343-349 (1949)

4. Dugan JJ, de Mayo P, Nisbet M, Robinson JR, Anchel M. Terpenoids. XIV The Constitution and Biogenesis of Marasmic Acid. J Am Chem Soc 88: 2838-2844 (1966)

5. Favre-Bonvin J, Gluchoff-Fiasson K, Bernillon J. Structure 
du stearyl-velutinal, sequiterpenoide naturel de lactarius velutinus bert. Tetrahedron Lett 23: 1907-1908 (1982)

6. Clericuzio M, Sterner O. Conversion of velutinal esters in the fruit bodies of Russula cuprea. Phytochemistry 45: 1569-1572 (1997)

7. Hansson T, Sterner O. Studies of the conversions of sesquiterpenes in injured fruit bodies of Lactarius vellereus.

A biomimetic transformation of stearoylvelutinal to isovelleral. Tetrahedron Lett 32: 2541-2544 (1991)

8. Heim J, Anke T, Mocek U, Steffan B, Steglich W. Antibiotics from basidiomycetes. XXIX: Pilatin, a new antibiotically active marasmane derivative from cultures of
Flagelloscypha pilatii Agerer. J Antibiot 41: 1752-1757 (1988)

9. Yaoita Y, Machida K, Kikuchi M. Structures of new marasmane sesquiterpenoids from Lactarius piperatus (Scop.: Fr.) S.F. Gray. Chem Pharm Bull 47: 894-896 (1999)

10. Wang XN, Wang F, Du JC, Ge HM, Tan RX, Liu JK. A new marasmane sesquiterpene from the basidiomycete Russula foetens (Pers.) Fr., Z Naturforsch 60b: 1065-1067 (2005)

11. Daniewski WM, Gumulka M, Ptaczynska K, Skibicki P, Krajewski J, Gluzinski P. Marasmane lactones from Lactarius vellereus. Phytochemistry 31: 913-915 (1992) 